\title{
Distributed, Hierarchical Clustering and Summarization in Sensor Networks*
}

\author{
Xiuli $\mathrm{Ma}^{1}$, Shuangfeng $\mathrm{Li}^{1}$, Qiong $\mathrm{Luo}^{2}$, Dongqing Yang ${ }^{1}$, and Shiwei Tang ${ }^{1}$ \\ ${ }^{1}$ School of Electronics Engineering and Computer Science, State Key Laboratory on \\ Machine Perception, Peking University, Beijing, China, 100871 \\ ${ }^{2}$ Department of Computer Science, The Hong Kong University of Science and Technology, \\ Clear Water Bay, Kowloon, Hong Kong \\ maxl@cis.pku.edu.cn, lishuangfeng@gmail.com, luo@cse.ust.hk, \\ \{dqyang, t.sw\} @ pku.edu.cn
}

\begin{abstract}
We propose DHCS, a method of distributed, hierarchical clustering and summarization for online data analysis and mining in sensor networks. Different from the acquisition and aggregation of raw sensory data, our method clusters sensor nodes based on their current data values as well as their geographical proximity, and computes a summary for each cluster. Furthermore, these clusters, together with their summaries, are produced in a distributed, bottom-up manner. The resulting hierarchy of clusters and their summaries facilitates interactive data exploration at multiple resolutions. It can also be used to improve the efficiency of data-centric routing and query processing in sensor networks. Our simulation results on real world data sets as well as synthetic data sets show the effectiveness and efficiency of our approach.
\end{abstract}

Keywords: Sensor networks, clustering, summarization.

\section{Introduction}

Many data-centric sensor network applications are not only interested in raw sensory readings of individual nodes, but are also interested in the patterns, outliers, and summaries of network-wide sensory data. For example, on the left of Fig.1 shows part of the deployment of a sensor network at the Intel Berkeley Lab together with a snapshot of the temperature sensor readings of individual nodes [3]. If we cluster the sensor nodes by their temperature readings and report the data range and average of each cluster, it gives a clear overview of the sensory data distribution as shown on the right of Fig.1. In addition to facilitating interactive data analysis, this kind of clustering and summary information is useful for datacentric routing and in-network query processing. Therefore, we study the problem of online clustering and summarization in sensor networks.

Since both clustering and summarization are computation-intensive tasks over a large amount of data, a natural solution is to conduct these tasks at a PC-grade base

This work is supported by the National Natural Science Foundation of China under Grant No.60473072, 60473051, and the National High Technology Development 863 Program of China under Grant No. 2006AA01Z230. 
station after the sensory readings are collected there. However, this centralized approach has two major drawbacks: one is timeliness and the other network power efficiency. In consideration of these drawbacks, we take a distributed approach to clustering and summarization in sensor networks.
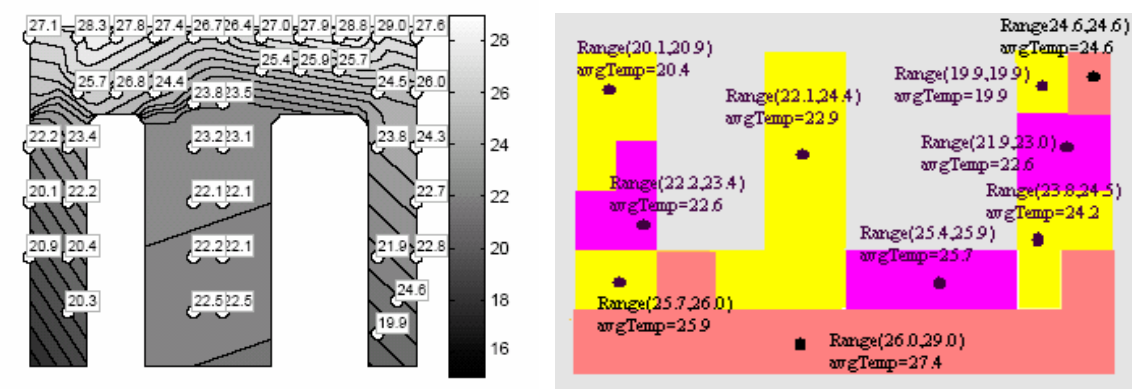

Fig. 1. Example of Clustering and Summarization

When considering distributing the clustering and summarization task to individual nodes in the network, we need to take into account the limited computing resources on each node and the multi-hop communication scheme of sensor networks. As a result, we first treat each sensor node as an initial cluster and then let geographically adjacent clusters gradually merge based on their readings. This bottom-up process is efficient because spatial correlations exist in real world sensory data so that computation and communication happens among proximate clusters mostly. In addition, summary information are also computed and maintained for the resulting hierarchy of clusters. As such, we call our method Distributed, Hierarchical Clustering and Summarization (DHCS).

Specifically, we propose a summary structure called Cluster Distribution Feature, or CDF, for each cluster. This feature includes both the data range and the statistical features of a cluster, and can be incrementally maintained along the hierarchy. Subsequently, we design a dissimilarity metric called expansion, to compute the dissimilarity between two clusters based on CDF. Furthermore, the computation complexity of both CDF and expansion is low and can be done efficiently in the network in a distributed manner. With these and other information, the DHCS algorithm clusters sensor nodes and computes summaries for clusters in a distributed, hierarchical manner.

Research on scalable clustering algorithms has been fruitful $[2,10,11]$. Unfortunately, these traditional clustering methods are infeasible in sensor networks, because they are mostly centralized. DHCS is distributed, thus can fully utilize nodes' computation ability. More, DHCS considers both data similarity and spatial proximity, whereas previous work has only considered data similarity. In recent years, there has been some work about clustering in sensor networks [1, 9]. However, they focus on network topology information. In comparison, DHCS is more data-centric and brings more opportunities for data reduction. Kotodis introduces the idea of snapshot queries towards data-centric sensor networks [7]. However, those representative nodes can only represent their one-hop neighbors, which limits the reduction ratio. 


\section{Preliminaries}

In this section, we first introduce the concept of Cluster Distribution Feature (CDF), then the dissimilarity metric expansion based on CDF, and finally the compact cluster.

\subsection{Cluster Distribution Feature}

Assume $N$ nodes are scattered in a region and each node can sense $d$ attributes, such as temperature and light. Assume the value of each attribute can be normalized by some normalization technique such as those in a recent survey [4]. Then, each node corresponds to a $d$-dimensional normalized data vector. A cluster of $N$ nodes corresponds to $N d$-dimensional data points, $\left\{X_{i}\right\}$, where $i=1,2, \ldots, N$.

A Cluster Distribution Feature summarizes the sensory data distribution information that we maintain about a cluster. It includes two components: Cluster Data Range and Cluster Feature.

Definition 1. Assume that there are $N$ nodes in a cluster, each of which can sense $d$ attributes. Let $\left\{X_{i}\right\}$, where $i=1,2, \ldots, N$, be the corresponding set of $N d$-dimensional data points. The Cluster Data Range (CDR) of the cluster is the smallest closed region in the data space into which all $X_{i}$ fall. The Spherical Cluster Data Range (SCDR) of the cluster is a tuple (Center, $R$ ), where Center is the center and $R$ is the radius of the smallest sphere into which all $X_{i}$ fall.

Intuitively, CDR provides a tight boundary for the data of the nodes in a cluster. For example, a circle or a rectangle in a $2 \mathrm{D}$ data space, a sphere or a cube in a $3 \mathrm{D}$ data space can be a CDR. In this paper we choose sphere for simplicity and intuitiveness. In the following of this paper, we use CDR and SCDR interchangeably.

We adopt Cluster Feature (CF) from Birch [10] to describe the statistical features of a cluster. Given the corresponding $N d$-dimensional data points of a cluster, $\left\{X_{i}\right\}$, where $i=1,2, \ldots, N$, the $\mathrm{CF}$ of the Cluster is a triple $\mathrm{CF}=(N, L S, s s)$, where $N$ is the number of data points in the cluster, $L S$ is the linear sum of the $N$ data points, and $s s$ is the square sum of the $N$ data points. CF facilitates the computation of the mean, deviation and other statistical features. More, it can be incrementally maintained [10].

Having the definitions of CDR and CF, we define the Cluster Distribution Feature.

Definition 2. Given $N$ nodes in a cluster, the Cluster Distribution Feature (CDF) of the cluster is a tuple $\mathrm{CDF}=\langle\mathrm{CDR}, \mathrm{CF}\rangle$.

Proposition 1. (CDF Additivity) Assume that the CDF of cluster $A$ is $\mathrm{CDF}_{A}$ $=<\left(\right.$ Center $\left._{A}, R_{A}\right),\left(N_{A}, L S_{A}, s s_{A}\right)>$, The CDF of cluster $B$ is $\mathrm{CDF}_{B}=<\left(\right.$ Center $\left._{B}, R_{B}\right),\left(N_{B}\right.$, $\left.L S_{B}, s s_{B}\right)>$, dist is the distance between Center $_{A}$ and Center $_{B}$. Then the CDF of cluster $C$ that is formed by merging $A$ and $B$, is the smallest sphere in the data space that can enclose the CDR of $A$ and the CDR of $B$. That is,

- when $\left(\right.$ dist $\left.+R_{B}\right) \leq R_{A}$, Center $_{C}=$ Center $_{A}, R_{C}=R_{A}$;

when $\left(R_{A}-R_{B}\right)<$ dist $<\left(R_{A}+R_{B}\right)$ or dist $\geq\left(R_{A}+R_{B}\right)$, Center C $_{C}$ is the middle point between Center $_{A}$ and Center $_{B}, R_{C}=\left(R_{A}+R_{B}+\right.$ dist $) / 2$.

- Addition of CF: the following additive law [10] holds:

$\left(N_{C}, L S_{C}, s s_{C}\right)=\left(N_{A}, L S_{A}, s s_{A}\right)+\left(N_{B}, L S_{B}, s s_{B}\right)=\left(N_{A}+N_{B}, L S_{A}+L S_{B}, s s_{A}+s s_{B}\right)$ 
From the CDF definition and the additivity proposition, we know that the CDF vectors of clusters can be stored and calculated incrementally as clusters are merged. These CDF vectors as summaries are not only efficient but also accurate for calculating the dissimilarity metric that we need for making clustering decisions in DHCS.

Next we define the dissimilarity metric between two clusters, expansion.

\subsection{Expansion}

Definition 3. Assume that the CDF of cluster $A$ is $\mathrm{CDF}_{A}=<\left(\right.$ Center $\left._{A}, R_{A}\right),\left(N_{A}, L S_{A}\right.$, $\left.s s_{A}\right)>$. The CDF of cluster $B$ is $\mathrm{CDF}_{B}=\left\langle\left(\right.\right.$ Center $\left._{B}, R_{B}\right),\left(N_{B}, L S_{B}, s s_{B}\right)>$. The CDF of cluster $C$, which is the cluster formed by merging $A$ and $B$, is $\mathrm{CDF}_{C}=<\left(\right.$ Center $\left._{C}, R_{C}\right)$, $\left(N_{C}, L S_{C}, s s_{C}\right)>$. Then expansion is the difference between $R_{C}$ and the larger one of $R_{A}$ and $R_{B}$. That is, expansion $=R_{C}-\max \left(R_{A}, R_{B}\right)$.

Essentially, expansion describes how much the CDR will expand after merging of two clusters. The smaller the expansion, the more similar the two clusters.

\subsection{Compact Cluster}

Assume that the normalized vector $\overrightarrow{e t}=\left(\Delta e_{1}, \Delta e_{2}, \ldots, \Delta e_{d}\right)$ is a predefined difference threshold, where $\Delta e_{i}$ is the maximum tolerable difference in the $i$-th attribute between any two nodes within a cluster. Assume that hopcount threshold is the maximum tolerable hop count between any two nodes within a cluster. The compact cluster is defined as follows:

Definition 4. Let $D$ be a cluster of $N$ nodes, each of which can sense $d$ attributes. Given the difference threshold $\overrightarrow{e t}=\left(\Delta e_{1}, \Delta e_{2}, \ldots, \Delta e_{d}\right)$ and hopcount threshold, a compact cluster $C$ is a non-empty subset of $D$ satisfying the following conditions:

- Similar sensory values: $\forall i, 1 \leq i \leq d,\left(R \times 2 \leq \Delta e_{i}\right)$, where $R$ is the radius of CDR of $C$;

- Geographical proximity. Two conditions must hold. First, any two nodes within $C$ can communicate with each other, possibly through intermediate nodes; if intermediate nodes are needed, they must be also in $C$. Second, the hop count between any two nodes in $C$ should be no greater than hopcount.

Different from traditional clustering methods, DHCS clusters nodes based on their current data values as well as their geographical proximity. Adjacency is defined as:

Definition 5. Assume $n_{i}\left(n_{j}\right)$ is a sensor node and cluster $C_{i} \quad\left(C_{j}\right)$ is a set of sensor nodes.

- Adjacent nodes: $n_{i}$ and $n_{j}$ are adjacent, or $n_{i}$ is a neighbor of $n_{j}$, if $n_{i}$ and $n_{j}$ can communicate with each other directly (within one hop).

- Adjacent clusters: $C_{i}$ and $C_{j}$ are adjacent, or $C_{i}$ is a neighbor of $C_{j}$, if there exist node $n_{p}$ in $C_{i}$ and node $n_{q}$ in $C_{j}, n_{p}$ and $n_{q}$ are adjacent.

DHCS produces compact clusters and their summaries, CDF vectors, in a bottom-up manner. Each compact cluster covers a local continuous region with similar sensory data. By partitioning sensors into several compact clusters and giving a summary for 
each cluster, DHCS divides the entire region into several sub-regions and keeps multiresolution summaries for each sub-region. These summaries are organized in trees.

Definition 6. A summary tree of a compact cluster $C$ is a tree structure of the sensor nodes in $C$ satisfying the following condition: the nodes in any sub-tree form a compact cluster $C_{i}$ with the root of the sub-tree being the cluster head for $C_{i}$, and storing the $\mathrm{CDF}$ of $C_{i}$.

\section{DHCS}

Given the difference and hopcount thresholds, DHCS produces compact clusters and their summaries in a distributed, bottom-up manner. Initially, each node treats itself as an active cluster. Then, similar adjacent clusters are merged into larger clusters round by round. In each round, each cluster will try to combine with its most similar adjacent cluster simultaneously. Two clusters can be merged only if both consider each other as the most similar neighbor. A compact cluster produced through merging must satisfy the thresholds. DHCS terminates when no merging happens any more. The final clusters, which cannot be merged any more, are called steady clusters.

In each round, each $\mathrm{CH}$ (short for cluster head) represents its cluster to coordinate with other clusters. In order for a $\mathrm{CH}$ to route to other $\mathrm{CH}$ s in its adjacent clusters efficiently, we maintain the adjacency information of a cluster in its $\mathrm{CH}$ and adapt $D S R$ (Dynamic Source Routing) [6] for DHCS. Thus, a $\mathrm{CH}$ keeps the CDF and adjacency information of its cluster. In DHCS, there are three kinds of nodes by their states:

- ACTIVE nodes: the $C H s$ of the active clusters;

- PASSIVE nodes: the nodes that are not $\mathrm{CH}$ of any cluster;

- STEADY nodes: the $C H s$ of the steady clusters.

Initially each node will be ACTIVE. ACTIVE nodes become PASSIVE or STEADY along the merging of clusters. DHCS terminates when there is no ACTIVE node. The STEADY nodes represent the final compact clusters.

Each round has four stages: advertising, inviting, accepting and merging. In the advertising stage, clusters exchange CDFs with neighbors. Then adjacent clusters may try to reach an agreement about merging by shaking hands in inviting and accepting stages. In the merging stage, new clusters are generated.

Next we describe the detailed operations in a round. Note that, each node has a globally unique hardware $I D[8]$, which we use as the node $I D$. Cluster $I D$ is defined as the $I D$ of its $C H$.

(1) Advertising CDF: Each ACTIVE node $n_{i}$ advertises the CDF of its cluster to the ACTIVE nodes of all its adjacent clusters simultaneously. After exchanging CDFs, each ACTIVE cluster determines the most similar neighbor by computing expansion based on CDFs. If more than one neighbor have the same expansion, we choose the one whose $I D$ is the largest. If a cluster cannot be merged any more given the thresholds, or if a cluster receives no messages, the state of its $\mathrm{CH}$ turns into STEADY.

(2) Sending invitation: For the purpose of coordination and avoiding redundant invitations, we take the following policy when sending invitations. Assume that $n_{i}$ considers $n_{j}$ as the most similar. $n_{i}$ sends an inviting message to $n_{j}$ only if (a) the cluster of $n_{i}$ 
has more nodes than $n_{j}$; or (b) the two clusters have the same number of nodes, and $n_{i}$ has the larger $I D$. Otherwise $n_{i}$ waits for invitations. After this stage, some ACTIVE nodes will receive several invitations. Note that $n_{i}$ will become the new $\mathrm{CH}$ if this merging succeed.

(3) Accepting an invitation: Suppose $n_{j}$ receives several invitations. Assume that $n_{j}$ considers $n_{i}$ as the most similar. If there is no invitation from $n_{i}, n_{j}$ will not accept any invitation; otherwise it will reply an accepting message to $n_{i}$. If $n_{j}$ accepts $n_{i}$ 's invitation, adjacency information of $n_{j}$ is piggybacked in the accepting message, and then $n_{j}$ sets its state as PASSIVE. By shaking hands, some pairs of adjacent clusters agree to merge.

(4) Merging clusters: If $n_{j}$ accepts $n_{i}$ 's invitation, a new cluster is generated by merging the nodes from the clusters of $n_{i}$ and $n_{j} . n_{i}$ becomes the new $C H$. The $I D$ of the new cluster is the $I D$ of node $n_{i}$. $n_{j}$ set its parent as $n_{i}$, while $n_{i}$ appends $n_{j}$ to its children. The $\mathrm{CHs}$ of these newly generated clusters finish the cluster merging by the following operations: (1) Compute the new CDF by the addition of the original two CDFs; and (2) Maintain the adjacency information of the new cluster.

After DHCS terminates, nodes' information about their parents, children and CDF will form several summary trees. Summary trees organize nodes considering data correlation as well as geographical proximity. They keep multi-resolution summaries to facilitate interactive data exploration at multiple resolutions. We leave the maintenance mechanism of summary trees for future work.

\section{Experiments}

We build a preliminary simulation environment to evaluate the performance of DHCS. First comes the effectiveness, then the efficiency. The following two datasets are used:

- The real geographical data set downloaded from Climatic Research Unit [12]. This website collects the climate data of 100 years of the entire world. We use a $30 \times 30$ grid taken from China's map covering the region with $24.5 \mathrm{~N}, 101.5 \mathrm{E}$ as the lower left corner located in Yunnan, and $39.5 \mathrm{~N}, 116.5 \mathrm{E}$ the upper right corner located in Beijing. Each grid cell corresponds to a half geographical degree and contains a point, which makes a total of 900 points. We extract the data of the average temperature in January 2002.

- Synthetic spatially-correlated data: In 4.2, we use the tool in [5] to generate larger synthetic datasets from a small $10 * 10$ sample of the real data set, to keep the same spatial correlation.

Let $w$ denote the network width. Nodes are arranged on a $w \times w$ grid, totally $w^{*} w$ nodes. $d$ is the transmission range. A large $d$ allows a large number of adjacent nodes for a node. $d t$ is the difference threshold. Assume that the communication is reliable.

We define two metrics to evaluate the quality of clusters: The reduction ratio is defined as $N / N_{C}$, where $N$ is the number of nodes and $N_{C}$ is the number of clusters. The average absolute deviation is the average absolute error when using the value of a cluster head to estimate those of any other nodes in the cluster. We consider only the impact of $d t$ by setting the hopcount threshold to be sufficiently large. 


\section{(1) Effectiveness of DHCS}

We vary $d t$ from 1 to 5 and $d$ from 1 to 3 . We can see in Fig.2, DHCS achieves the reduction ratios of 10 to 50 for various parameters. The reduction ratio will increase with the increase of $d t$ and $d$, due to the increase of cluster size. Fig. 3 shows that the average absolute deviation is significantly smaller than $d t$ used, about 1/6 of $d t$. Additionally, the deviation has little correlation with $d$. Both the reduction ratio and the average absolute deviation are mainly influenced by data correlation and $d t$.

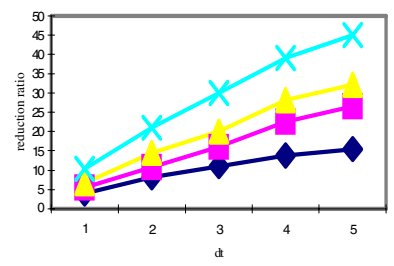

Fig. 2. Reduction ratio

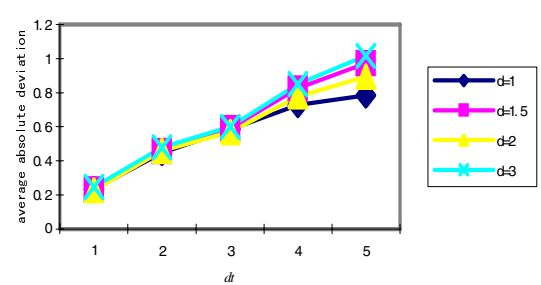

Fig. 3. Average absolute deviation

\section{(2) Efficiency of DHCS}

The most important metric for sensor networks is power efficiency. Therefore, we evaluate the efficiency of DHCS by the number of messages transmitted. We generate $w^{*} w$ datasets with $w$ varied from 40 to 160 , with step 20 . For the centralized clustering, the main cost is in collecting data.

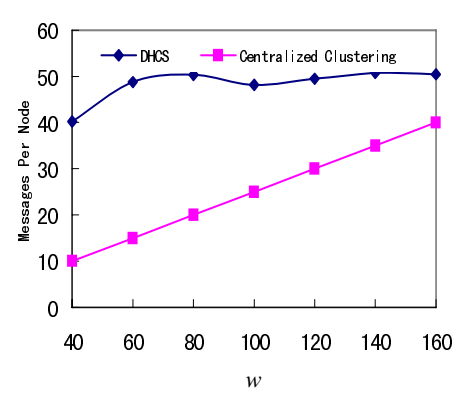

(a) $d=2$

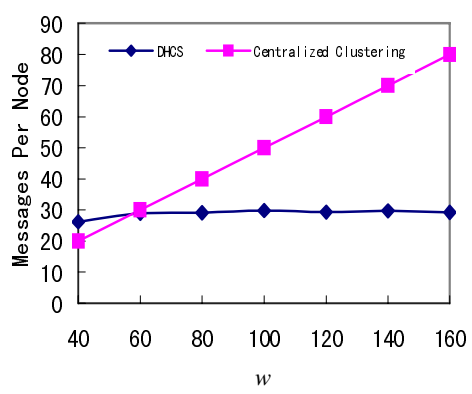

(b) $d=1$

Fig. 4. DHCS vs. Centralized Clustering $(d t=3)$

Suppose the sink node resides at the center of the upper side of a $w^{*} w$ square, then the depth of the routing tree is about $w / d$ and on average $w /(2 d)$ messages per node for collecting all data to the sink. We use $w /(2 d)$ to represent the cost of centralized clustering.

When $d$ is 2 in Fig. 4(a), DHCS is worse than centralized clustering. The main reason is that only two clusters are merged at a time in DHCS, which slows down the convergence of clustering. Fortunately, this downside is compensated by the scalability of DHCS. As shown in the figures, DHCS will eventually outperform centralized clustering given a sufficiently large network, although we cannot finish the 
experiment in larger network limited by our simulation platform. Fig. 4(b) shows that, when $d$ is 1 , the cost of DHCS is rather stable, about 30 messages per node. In contrast, the cost of the centralized clustering increases linearly with $w$, about 80 messages when $w$ is 160 . The larger the network is, the better DHCS performs.

\section{Conclusion}

We propose DHCS, a method of distributed, hierarchical clustering and summarization for sensor networks. DHCS clusters nodes based on their current data values as well as their geographical proximity in a distributed, bottom-up manner. The resulting hierarchy of clusters and their summaries can provide quick overviews about the network and facilitate interactive data exploration at multiple resolutions.

Future work includes extending DHCS to merging more than two clusters at a time, designing maintenance mechanisms for the clustering and summary information, and evaluating DHCS in real sensor networks.

\section{References}

1. S. Bandyopadhyay and E. J. Coyle. An Energy Efficient Hierarchical Clustering Algorithm for Wireless Sensor Networks. INFOCOM 2003.

2. M. M. Breunig, H. Kriegel, P. Kroger, and J. Sander. Data Bubbles: Quality Preserving Performance Boosting for Hierarchical Clustering. SIGMOD 2001.

3. C. Guestrin, P. Bodik, R. Thibaux, M. Paskin, and S. Madden. Distributed Regression: An Efficient Framework for Modeling Sensor Network Data. IPSN 2004.

4. J. Han and M. Kamber. Data Mining: Concepts and Techniques. China Machine Press, 2001.

5. A. Jindal and K. Psounis. Modeling Spatially-Correlated Sensor Network data. SECON 2004.

6. D. B. Johnson and D. A. Maltz. Dynamic Source Routing in Ad-hoc Wireless Networks. Mobile Computing, Kluwer Academic Publishers, pp. 153-181, 1996.

7. Y. Kotidis. Snapshot Queries: Towards Data-Centric Sensor Networks. ICDE, 2005.

8. S. Madden, M. J. Franklin, J. M. Hellerstein, and W. Hong. Tag: A Tiny Aggregation Service for ad hoc Sensor Networks. OSDI 2002.

9. O. Younis and S. Fahmy. Distributed Clustering in Ad-hoc Sensor Networks: A Hybrid, Energy-efficient Approach. INFOCOM 2004.

10. T. Zhang, R. Ramakrishnan, and M. Livny. BIRCH: An efficient data clustering method for very large databases. SIGMOD 1996.

11. T. Zhou, R. Ramakrishnan, and M. Livny. Data Bubbles for Non-Vector Data: Speedingup Hierarchical Clustering in Arbitrary Metric Spaces. VLDB 2003.

12. http://www.cru.uea.ac.uk/ 